\title{
Characterisation of Microstructural Changes during the Hydration of Pharmaceutical Tablets using Terahertz Pulsed Imaging
}

\author{
Daniel Markl, Samy Yassin, Parry Wang and J. Axel Zeitler \\ Department of Chemical Engineering and Biotechnology, University of Cambridge, Pembroke Street, Cambridge, UK, CB2 3RA
}

\begin{abstract}
Terahertz pulsed imaging (TPI) is used to characterise microstructural changes during the hydration of pharmaceutical tablets. In particular, terahertz reflection measurements provide quantitative information about the liquid penetration, swelling and the formation of cracks. $\mathrm{X}$-ray computed microtomography $(\mathrm{X} \mu \mathrm{CT})$ is employed to characterise the microstructure of the dry powder compact in terms of porosity, equivalent volume diameter and length of the pores. Both $\mathrm{X \mu CT}$ and TPI can provide valuable information about the impact of the microstructure on the performance of drugs.
\end{abstract}

\section{INTRODUCTION AND BACKGROUND}

$\mathbf{P}$ HARMACEUTICAL tablets are still the predominant form to administer active pharmaceutical ingredients (APIs) to the patient. Tablets are typically powder compacts consisting of several different excipients in addition to the API. Excipients are added to a formulation in order to achieve the desired fill weight of a dosage form, to improve the processability or to affect the drug release in the body. Beside the chemical difference between the different excipients and APIs, they highly vary in terms of physical properties, such as particle size, morphology or hygroscopicity. All these parameters directly impact the microstructure of the pharmaceutical tablet, which further highly affects the performance of the drug in the patient's body. Moreover, these complex porous systems are primarily formulated on the basis of empirical knowledge and the behaviour of the powder compact when it comes in contact with human gastric fluids is only poorly understood.

However, significant advances in analytical techniques during the last years enabled the quantitative investigation of changes in the microstructure during the hydration of a pharmaceutical tablet. In particular, one of the most promising techniques in this field is terahertz pulsed imaging (TPI). Most of the excipients are transparent to terahertz radiation, whereas interfaces from substructures within a tablet lead to a contrast in the terahertz measurements due to a change in their refractive index [1]. The liquid penetrating the powder compact changes the refractive index of the wetted material and thus enables the tracking of the penetration front (transient from wetted to dry material) by means of $\mathrm{THz}$ reflection technology [1], [2]. This study demonstrates how TPI can be used to characterise cracks formed on the tablet surface and within the powder compact during hydration and subsequent crack propagation during swelling of the tablet beside investigating

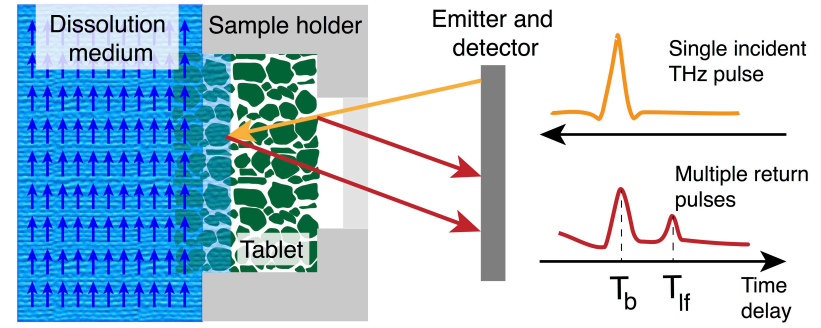

Fig. 1. Experimental setup for measuring the hydration of pharmaceutical powder compacts. The $\mathrm{THz}$ pulse at $T_{l f}$ corresponds to the interface between wetted and dry medium. The pulse at $T_{f}$ represents the back face of the tablet.

the liquid ingress and the swelling of the tablet.

\section{RESULtS}

A custom made flow-through cell was developed to monitor the liquid ingress in a pharmaceutical powder compact in conjunction with a commercial TPI system (Imaga 2000, Teraview Ltd, Cambridge, UK) [1], [2]. This setup exposes the liquid to a single face of the tablet with a diameter of $10 \mathrm{~mm}$ and a thickness of $1.5 \mathrm{~mm}$. The tablets were compacted using a compaction simulator (Pheonix Calibrations, Pheonix, USA).

The liquid penetrating the powder compact induces changes of the refractive index and thus the penetration front can be tracked by means of $\mathrm{THz}$ reflection technology as schematically illustrated in Fig. 1. Tracking $T_{l f}$ during the hydration of a powder compact facilitates the analysis of liquid penetration kinetics (see Fig 3).

During powder compaction, particles are deformed and inter-particulate bonds are forged. The breakage of these bonds during the hydration process leads to cracks on both the tablet surface and within the tablet matrix that can be detected by additional reflections in the terahertz time-domain waveforms (see Fig. 3). Swelling of the powder particles strains the tablet structure forming micro-cracks, which in turn increases the pore space in which water can enter. This process accelerates tablet hydration and subsequent hydration of the tablet. Therefore, the pore structure of the powder compact has a huge impact on the drug performance. Porous media (i.e. powder compacts) can be characterised using X-ray computed microtomography $(\mathrm{X} \mu \mathrm{CT})$ enabling the extraction of 


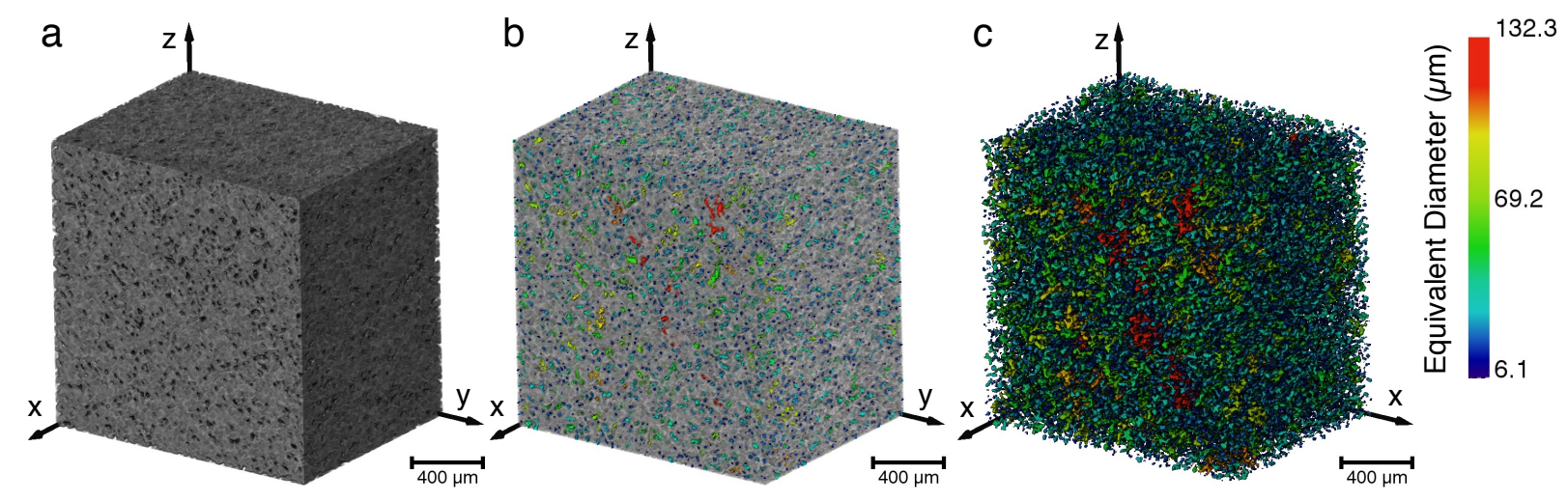

Fig. 2. 3D renderings of $\mathrm{X} \mu \mathrm{CT}$ data representing a subvolume $\left(1.73 \times 1.73 \times 1.34 \mathrm{~mm}^{3}\right)$ of a pharmaceutical tablet containing 95 wt\% microcrystalline cellulose (MCC, PH102, FMC Biopolymers, Philadelphia, USA) and $5 \mathrm{wt} \%$ croscarmellose sodium. The solid material is shown in (a). The pore space was extracted from the subvolume and is visualised in (c). (b) depicts both the solid matrix and the pore space.

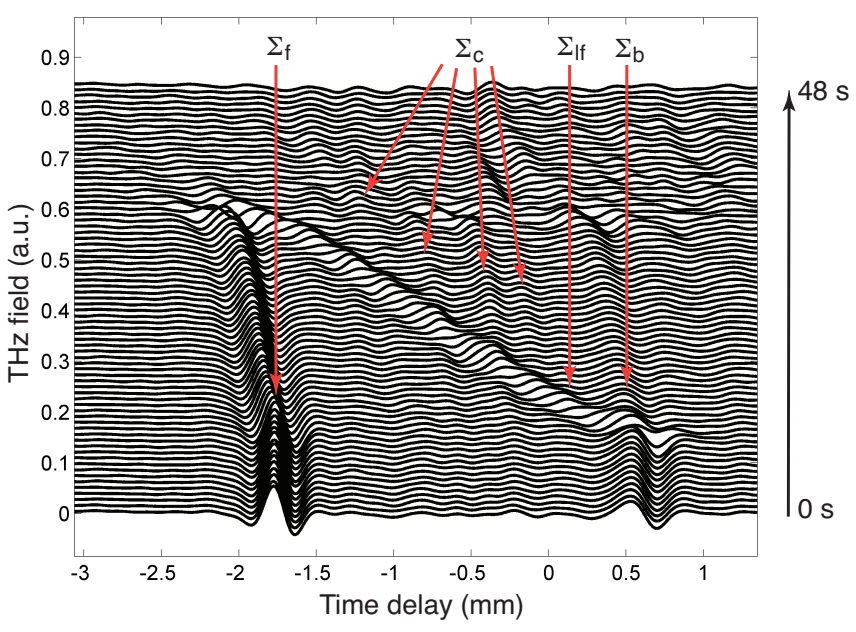

Fig. 3. Deconvolved time-domain waveforms (each line is offset by 0.01 a.u.) showing the hydration of a pharmaceutical tablet consisting of $44 \%$ lactose anhydrous (Tablettose 100, Meggle, Wasserburg, Germany), 30\% microcrystalline cellulose (MCC PH102, FMC Biopolymers, Philadelphia, USA), $20 \%$ paracetamol, $5 \%$ croscarmellose sodium, $1 \%$ magnesium stearate with $10 \%$ porosity. $\Sigma_{f}$ (related to $T_{l f}$ in Fig. 1) corresponds to the front surface of the tablet, $\Sigma_{b}$ corresponds to the back surface of the tablet, $\Sigma_{l f}$ (corresponding to $T_{l f}$ in Fig. 1) corresponds to the hydration front and $\Sigma_{c}$ corresponds to the formation of cracks within the tablet matrix due to the strain caused by the hydration.

the entire pore space. A pharmaceutical tablet was analysed using a SkyScan 1172 high-resolution XCT scanner (Bruker, Antwerp, Belgium), which utilises a cone beam geometry in combination with a 2D array detector. Fig. 2 illustrates $\mathrm{X} \mu \mathrm{CT}$ data, which was imaged at an isotropic voxel resolution of $4.94 \mu \mathrm{m}$. X $\mu \mathrm{CT}$ data yielded a porosity, average equivalent volume diameter and average pore length of $8.0 \%, 17.2 \pm$ $10.8 \mu \mathrm{m}$ and $35.1 \pm 34.5 \mu \mathrm{m}$, respectively. The porosity of the tablet was also measured by helium pycnometry (Accupyc 1330, Micrometrics, Norcross, USA) yielding a porosity of $10.2 \pm 0.7 \%$.

During the hydration of the tablet, the pore space changes and once a region of the tablet is fully hydrated individual polymer particles start to swell. This swelling puts a strain on the tablet matrix and cracks begin to form once this strain exceeds the inter-particulate strength of the tablet. Combining the information from the terahertz reflection measurements about liquid penetration, swelling and the formation of cracks provides fundamental information about the microstructural behaviour of these complex powder compacts.

\section{CONCLUSION}

TPI is a very promising technology to quantitatively study the hydration process and to relate the extracted information to material properties and process configurations. The results from TPI will be further related to pore structure parameters extracted from $\mathrm{X} \mu \mathrm{CT}$ measurements. Clearly, advances in understanding mass transport mechanisms will also be of central importance for many other application areas outside the pharmaceutical industry.

\section{ACKNOWLEDGMENT}

The authors would like to thank the EPSRC for funding and D.J. Goodwin and A. Anderson from GSK for access to a compaction simulator, materials and discussions.

\section{REFERENCES}

[1] S. Yassin, D. J. Goodwin, A. Anderson, J. Sibik, D. I. Wilson, L. F. Gladden, and J. A. Zeitler, "The Disintegration Process in Microcrystalline Cellulose Based Tablets, Part 1: Influence of Temperature, Porosity and Superdisintegrants," Journal of Pharmaceutical Sciences, vol. 104, no. 10, pp. 3440-3450, 2015.

[2] S. Yassin, K. Su, H. Lin, L. F. Gladden, and J. A. Zeitler, "Diffusion and Swelling Measurements in Pharmaceutical Powder Compacts Using Terahertz Pulsed Imaging," Journal of Pharmaceutical Sciences, vol. 104, no. 5, pp. 1658-1667, 2015. 